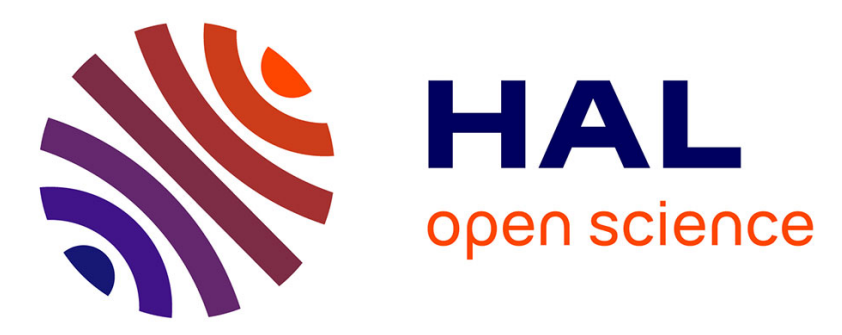

\title{
Analyse dimensionnelle de l'équation de Navier et application à la théorie des plaques minces
}

Olivier Millet, Aziz Hamdouni, Alain Cimetière, Khalid Elamri

\section{To cite this version:}

Olivier Millet, Aziz Hamdouni, Alain Cimetière, Khalid Elamri. Analyse dimensionnelle de l'équation de Navier et application à la théorie des plaques minces. Journal de Physique III, 1997, 7 (10), pp.1909-1925. 10.1051/jp3:1997232 . jpa-00249690

\section{HAL Id: jpa-00249690 https://hal.science/jpa-00249690}

Submitted on 1 Jan 1997

HAL is a multi-disciplinary open access archive for the deposit and dissemination of scientific research documents, whether they are published or not. The documents may come from teaching and research institutions in France or abroad, or from public or private research centers.
L'archive ouverte pluridisciplinaire HAL, est destinée au dépôt et à la diffusion de documents scientifiques de niveau recherche, publiés ou non, émanant des établissements d'enseignement et de recherche français ou étrangers, des laboratoires publics ou privés. 


\title{
Analyse dimensionnelle de l'équation de Navier et application à la théorie des plaques minces
}

\author{
Olivier Millet, Aziz Hamdouni $\left(^{*}\right)$, Alain Cimetière et Khalid Elamri \\ L3 M A, Université de Poitiers et ENSMA, SP2MI Téléport 2, 86960 Futuroscope Cedex, France
}

(Reçu le 3 mars 1997, révisé le 4 juin 1997, accepté le 26 juin 1997)

PACS.46 30.cn - Static elasticity

\begin{abstract}
Résumé. - L'analyse dimensionnelle de l'équation de Navier fait apparaître de façon naturelle des nombres sans dımension caractéristiques de la nature physique du problème. Ces nombres sans dimension permettent de déterminer le domaine de validité du modèle de Kirchhoff-Love. Cette nouvelle approche ne nécessite aucune hypothèse a pron de changement d'échelle pour mettre en œuvre la méthode des développements asymptotıques.
\end{abstract}

\begin{abstract}
The dimensional analysis of the three-dimensional Navier equation leads to nondimensional numbers which reflect the physical problem. Thanks to these numbers, the twodimensional Kirchhoff-Love model can be proved valid for level forces of $\varepsilon^{4}$ order To be run, the asymptotic method developped in this paper, needs no a przorn assumption on the changing of scales.
\end{abstract}

\section{Introduction}

La première justification rigoureuse des modèles bidimensionnels linéaire et non linéaire de plaques minces a été obtenue par P.G. Ciarlet et P. Destuynder $[2-5,13]$ à la fin des années 70 grâce à la technique des développements asymptotıques appliquée à une formulation variationnelle mixte en contraintes et déplacements du problème tridimensionnel initial. Le cadre fonctionnel utilisé est particulièrement bien adapté pour démontrer certaıns résultats d'existence, d'unicité et de convergence. Par la suite, une approche variationnelle en déplacements seuls, variante de l'approche en contraintes et déplacements, fut proposée dans $[6,17]$. On notera également que la technique des développements asymptotiques a été appliquée avec succès dans le cas des plaques non homogènes [1] et pour des comportements élastiques non linéaires $[11,12]$. Parallèlement, ces travaux furent étendus à certains modèles de coques par P. Destuynder [13], P G. Ciarlet [7-10] et E. Sanchez-Palencia [18-20,22].

Cependant deux problèmes subsistent encore à notre connaissance dans la justification du modèle bidimensionnel linéaire de plaque par développements asymptotiques, à savoir :

1 - le choix d'hypothèses a prıorı de changements d'échelle sur le déplacement. Ces changements d'échelle déterminent l'ordre de grandeur du rapport entre les échelles de déplacement normal et de déplacement tangentiel. Notons qu'une tentative de justification de ces changements d'échelle a été proposée dans [15].

$\left(^{*}\right)$ Auteur auquel doit être adressée la correspondance (e-mail : hamdouA@l3ma.univ-poitiers.fr)

(C) Les Éditions de Physique 1997 
2- l'interprétation physique difficile des changements d'échelle portant sur les efforts. En effet, ces changements d'échelle rendent l'effort volumique s'exerçant sur la plaque dépendant de son épaisseur $2 h$. Or dans les problèmes usuels d'élasticité, la seule force volumique agissant est celle associée à la pesanteur $(\rho g)$ qui est indépendante de l'épaisseur.

Nous proposons ici une nouvelle justification par méthodes asymptotiques du modèle linéaire de plaques minces permettant de résoudre les deux problèmes précités. Cette approche, qui s'inscrit dans la lignée des travaux de Goldenveizer [14], est basée sur une adımensionnalisation de l'équation de Navier. Elle ne nécessite aucune hypothèse a pror sur l'ordre de grandeur du rapport entre les échelles de déplacement normal et de déplacement tangentiel.

L'adimensionnalisation de l'équation de Navier permet de faire apparaître de façon naturelle des nombres sans dimension gouvernant le problème d'élastıcité linéaire.

L'interprétation physique de ces nombres sans dimension permet alors de les relier au facteur de forme $\varepsilon=\frac{h}{L}$ (rapport de la demi-épaisseur sur le diamètre de la surface moyenne de la plaque).

Pour une plaque mince, $\varepsilon$ étant petit, on met en œuvre la technique des développements asymptotiques avec $\varepsilon$ comme petit paramètre. Pour les niveaux d'efforts usuellement rencontrés en mécanique, on aboutit au modèle bidimensionnel de Kirchhoff-Love de plaque mince dont le domaine de validité se trouve ainsi précisé grâce aux nombres adimensionnels introduits.

Il est ensuite possible, sans faire aucune hypothèse a pron, de déterminer l'expression des contraintes avant de revenir aux variables physiques.

\section{Position du problème}

Dans tout ce qui suit, les indices grecs seront supposés varier dans $\{1,2\}$ et les indices latins dans $\{1,2,3\}$. On supposera également choisies une fois pour toutes une origine $O$ ainsi qu'une base orthonormée $\left(e_{1}, e_{2}, e_{3}\right)$ dans l'espace euclidien de dimension trois qui sera ensuite identifié à $\mathbb{R}^{3}$. On indexera par une étoile $(*)$ toutes les grandeurs dimensionnelles et on adoptera les notations suivantes:

- $\mathrm{Grad}^{*}, \mathrm{Div}^{*}$ et $\Delta_{3}^{*}$ désigneront respectivement le gradient, le vecteur divergence et le laplacien tridimensionnels,

- $\operatorname{grad}^{*}, \operatorname{div}^{*}$ et $\Delta^{*}$ le gradient, le vecteur divergence et le laplacien bidimensionnels.

Soit $\omega^{*}$ un ouvert borné connexe de $\mathbb{R}^{2}$ contenu dans le plan $\left(O, e_{1}, e_{2}\right)$, de diamètre $L$, de point courant $x^{*}=\left(x_{1}^{*}, x_{2}^{*}\right)$ et de frontière $\gamma_{0}^{*}$ régulière. On définit les ensembles :

$$
\begin{gathered}
\left.\Omega^{*}=\omega^{*} \times\right]-h, h[ \\
\Gamma_{0}^{*}=\gamma_{0}^{*} \times[-h, h] ; \quad \Gamma_{ \pm}^{*}=\bar{\omega}^{*} \times\{ \pm h\}
\end{gathered}
$$

La frontière $\partial \Omega^{*}$ est alors la réunion de la frontière latérale $\Gamma_{0}^{*}$ et des faces supérieure et inférieure $\Gamma_{ \pm}^{*}$. On notera $X^{*}=\left(x^{*}, x_{3}^{*}\right)$ le point courant de $\Omega^{*}$.

Considérons alors une plaque mince d'épaisseur $2 h$ et de surface moyenne $\omega^{*}$, occupant le domaine $\Omega^{*}$ de $\mathbb{R}^{3}$ dans sa configuration non déformée, supposée encastrée sur son bord latéral $\Gamma_{0}^{*}$, et soumise aux efforts volumiques $\left(f_{2}^{*}\right): \bar{\Omega}^{*} \rightarrow \mathbb{R}^{3}$ et surfaciques $\left(g_{\imath}^{* \pm}\right): \Gamma_{ \pm}^{*} \rightarrow \mathbb{R}^{3}$.

On notera

$$
f^{*}=f_{\alpha}^{*} e_{\alpha}+f_{3}^{*} e_{3}=f_{\mathrm{t}}^{*}+f_{3}^{*} e_{3} \quad \text { et } \quad g^{* \pm}=g_{\alpha}^{* \pm} e_{\alpha}+g_{3}^{* \pm} e_{3}=g_{\mathrm{t}}^{* \pm}+g_{3}^{* \pm} e_{3} .
$$

Nous ferons dans tout ce qui suit l'hypothèse de plaque mince $(h<<L)$. 
Sous l'action des efforts extérieurs la plaque se déforme et l'inconnue du problème est alors le déplacement $U^{*}: \bar{\Omega}^{*} \rightarrow \mathbb{R}^{3}$ tel qu'un point matériel occupant initialement la position $X^{*} \in \bar{\Omega}^{*}$ occupe dans la configuration déformée la position $X^{*}+U^{*}\left(X^{*}\right)$. La plaque étant supposée encastrée sur son bord latéral $\Gamma_{0}^{*}$, on a :

$$
U^{*}=0 \quad \operatorname{sur} \Gamma_{0}^{*}
$$

Dans le cadre de l'élasticité linéaire en petites déformatıons et en petits déplacements, les équations d'équlibre s'écrivent :

$$
\left\{\begin{array}{c}
\operatorname{Div}{ }^{*}\left[\lambda \operatorname{Tr} e^{*}\left(U^{*}\right) I+2 \mu e^{*}\left(U^{*}\right)\right]=-f^{*} \operatorname{dans} \Omega^{*} \\
U^{*}=0 \quad \operatorname{sur} \Gamma_{0}^{*} \\
\left\{\lambda \operatorname{Tr} e^{*}\left(U^{*}\right) I+2 \mu e^{*}\left(U^{*}\right)\right\} n=g^{*} \text { sur } \Gamma_{ \pm}^{*}
\end{array}\right.
$$

où

$$
e^{*}\left(U^{*}\right)=\frac{1}{2}\left(\operatorname{Grad}^{*} U^{*}+\overline{\operatorname{Grad}^{*} U^{*}}\right)
$$

représente le tenseur linéarisé des déformations, $n$ la normale unitaire extérieure à $\partial \Omega^{*}, \lambda$ et $\mu$ les constantes de Lamé du matériau, la barre étant l'opérateur de transposition.

\section{Adimensionnalisation des équations de Navier}

Les équations d'équilibre précédentes conduisent naturellement à l'équation de Navier qui s'écrit.

$$
\left\{\begin{array}{c}
(\lambda+\mu) \operatorname{Grad}^{*}\left(\operatorname{Div}^{*} U^{*}\right)+\mu \Delta_{3} U^{*}=-f^{*} \quad \operatorname{dans} \Omega^{*} \\
U^{*}=0 \quad \text { sur } \Gamma_{0}^{*} \\
\left\{\lambda \operatorname{Tr} e\left(U^{*}\right) I+2 \mu e^{*}\left(U^{*}\right)\right\} n=g^{*} \text { sur } \Gamma_{ \pm}^{*} .
\end{array}\right.
$$

En décomposant maintenant le déplacement $U^{*}$ suivant une composante tangentielle et une composante normale

$$
U^{*}=V^{*}+u_{3}^{*} e_{3}
$$

les équations de Navier deviennent :

$$
\begin{array}{ll}
(\lambda+\mu) \operatorname{grad}^{*}\left(\operatorname{div}^{*} V^{*}\right)+\mu \Delta^{*} V^{*}+(\lambda+\mu) \operatorname{grad}^{*} \frac{\partial u_{3}^{*}}{\partial x_{3}^{*}}+\mu \frac{\partial^{2} V^{*}}{\partial x_{3}^{2 *}} & =-f_{\mathrm{t}}^{*} \\
(\lambda+\mu) \frac{\partial}{\partial x_{3}^{*}} \operatorname{div}^{*} V^{*}+\mu \Delta^{*} u_{3}^{*}+(\lambda+2 \mu) \frac{\partial^{2} u_{3}^{*}}{\partial x_{3}^{2 *}} & =-f_{3}^{*} .
\end{array}
$$

Les conditions aux limites sur les faces supérieure et inférieure $\Gamma_{ \pm}^{*}$ sont données par :

$$
\begin{gathered}
\mu\left(\operatorname{grad}^{*} u_{3}^{*}+\frac{\partial V^{*}}{\partial x_{3}^{*}}\right)= \pm g_{\mathrm{t}}^{* \pm} \quad \operatorname{sur} \bar{\omega}^{*} \times\{ \pm h\} \\
(\lambda+2 \mu) \frac{\partial u_{3}^{*}}{\partial x_{3}^{*}}+\lambda \operatorname{div} V^{*}= \pm g_{3}^{* \pm} \quad \operatorname{sur} \bar{\omega}^{*} \times\{ \pm h\} .
\end{gathered}
$$


Effectuons alors l'adimensionnalisation suivante sur les données physiques et les inconnues du problème :

$$
\begin{aligned}
& V=\frac{V^{*}}{V_{\mathrm{r}}} \quad u_{3}=\frac{u_{3}^{*}}{u_{3 \mathrm{r}}} \quad x_{\alpha}=\frac{x_{\alpha}^{*}}{L} \quad x_{3}{ }^{\prime}=\frac{x_{3}^{*}}{h} \\
& f_{3}=\frac{f_{3}^{*}}{f_{3 \mathrm{r}}} \quad g_{3}^{ \pm}=\frac{g_{3}^{* \pm}}{g_{3 \mathrm{r}}} \quad f_{\mathrm{t}}=\frac{f_{\mathrm{t}}^{*}}{f_{\mathrm{tr}}} \quad g_{\mathrm{t}}^{ \pm}=\frac{g_{\mathrm{t}}^{* \pm}}{g_{\mathrm{tr}}} .
\end{aligned}
$$

où les grandeurs indexées en $r$ sont les grandeurs de référence du problème. Les nouvelles variables (sans étoile) qui apparaissent alors sont adimensionnelles.

L'objectif étant ici d'obtenir un modèle bidimensionnel valable en petits déplacements et petites déformations, on choisit dans un premier temps la même échelle de référence $u_{3 \mathrm{r}}=V_{\mathrm{r}}=h$ pour le déplacement normal et le déplacement transverse. Notons maintenant grad, div et $\Delta$ le gradient, le vecteur divergence et le laplacien bidimensionnels définis sur $\omega$ (par rapport aux variables adimensionnelles $x_{\alpha}$ ).

Les équations de Navier (1) s'écrivent alors dans $\Omega=\omega \times]-1,1[$ :

$$
\begin{array}{ll}
\varepsilon^{2}[(1+\beta) \operatorname{grad}(\operatorname{div} V)+\Delta V]+(1+\beta) \varepsilon \operatorname{grad}\left(\frac{\partial u_{3}}{\partial x_{3}}\right)+\frac{\partial^{2} V}{\partial x_{3}^{2}}=-\mathcal{F}_{\mathrm{t}} f_{\mathrm{t}} \\
\varepsilon^{2} \Delta u_{3}+(1+\beta) \varepsilon \frac{\partial}{\partial x_{3}} \operatorname{div} V+(2+\beta) \frac{\partial^{2} u_{3}}{\partial x_{3}^{2}} & =-\mathcal{F}_{3} f_{3}
\end{array}
$$

avec les conditions aux limites ·

$$
\begin{array}{llll}
\varepsilon \operatorname{grad} u_{3}+\frac{\partial V}{\partial x_{3}} & = \pm \mathcal{G}_{\mathrm{t}} g_{\mathrm{t}}^{ \pm} & \text {sur } & \bar{\omega} \times\{ \pm 1\} \\
(2+\beta) \frac{\partial u_{3}}{\partial x_{3}}+\beta \varepsilon \operatorname{div} V & = \pm \mathcal{G}_{3} g_{3}^{ \pm} & \text {sur } & \bar{\omega} \times\{ \pm 1\}
\end{array}
$$

où l'on a posé : $\quad \beta=\frac{\lambda}{\mu}$

On voit donc apparaître naturellement cinq nombres sans dimension :

$$
\varepsilon=\frac{h}{L} \quad \mathcal{F}_{3}=\frac{h f_{3 \mathrm{r}}}{\mu} \quad \mathcal{F}_{\mathrm{t}}=\frac{h f_{\mathrm{tr}}}{\mu} \quad \mathcal{G}_{3}=\frac{g_{3 \mathrm{r}}}{\mu} \quad \mathcal{G}_{\mathrm{t}}=\frac{g_{\mathrm{tr}}}{\mu}
$$

i) le rapport de forme $\varepsilon=\frac{h}{L}$, rapport entre l'épaisseur et le diamètre de $\omega^{*}$ est une donnée du problème. Dans le cadre des plaques minces $\varepsilon$ est un petit paramètre.

ii) les rapports de forces $\mathcal{F}_{\mathrm{t}}=\frac{h f_{\mathrm{tr}}}{\mu}, \mathcal{F}_{3}=\frac{h f_{3 \mathrm{r}}}{\mu}$ et $\mathcal{G}_{\mathrm{t}}=\frac{g_{\mathrm{tr}}}{\mu}, \mathcal{G}_{3}=\frac{g_{3 \mathrm{r}}}{\mu}$ représentent respectivement le rapport entre la résultante des efforts volumiques sur l'épaisseur (respectivement entre les efforts surfaciques) et $\mu$ regardé comme contrainte de référence. Ces nombres ne font intervenir que des grandeurs connues et doivent donc être considérés comme des données du problème. 


\section{Hypothèse mono-échelle}

Dans le but de se ramener à un problème mono-échelle pour des raisons de simplification, il convient de définir une variable de référence et de relier ensute les autres à cette dernière. Nous choisirons ici $\varepsilon$ comme paramètre de référence et nous exprimerons les autres nombres sans dimension en fonction de $\varepsilon$.

Les efforts extérieurs constituent une des données essentielles du problème. Dans le cadre d'une hypothèse mono-échelle, il convient de relier les rapports de forces au facteur de forme $\varepsilon$ ou de façon équivalente de se donner l'ordre de grandeur des efforts appliqués. Il est tout d'abord possible de relier $\mathcal{F}_{\mathrm{t}}$ à $\mathcal{G}_{\mathrm{t}}$ et $\mathcal{F}_{3}$ à $\mathcal{G}_{3}$ afin que tous les efforts interviennent au même ordre dans l'équation d'équilibre globale de la plaque. En effet l'équation d'équilibre globale s'écrit :

$$
\int_{\Omega^{*}} f^{*} \mathrm{~d} x^{*}+\int_{\Gamma^{*+}} g^{*+} \mathrm{d} \Gamma^{*}+\int_{\Gamma^{*-}} g^{*-} \mathrm{d} \Gamma^{*}+\int_{\Gamma_{0}^{*}} H^{*} \mathrm{~d} \Gamma^{*}=0
$$

où $H^{*}=H_{t}^{*}+H_{3}^{*} e_{3}$ représente les efforts d'encastrement. Après adimensionnalisation et projection respective sur $\omega$ et suivant la direction $e_{3}$, on obtient :

$$
\begin{aligned}
& h f_{\mathrm{tr}} \int_{\Omega} f_{\mathrm{t}} \mathrm{d} x+g_{\mathrm{tr}}\left[\int_{\Gamma^{+}} g_{\mathrm{t}}^{+} \mathrm{d} \Gamma+\int_{\Gamma^{-}} g_{\mathrm{t}}^{-} \mathrm{d} \Gamma\right]+\frac{H_{\mathrm{tr}} h}{L} \int_{\Gamma_{0}} H_{\mathrm{t}} \mathrm{d} s \mathrm{~d} x_{3}=0 \\
& h f_{3 \mathrm{r}} \int_{\Omega} f_{3} \mathrm{~d} x+g_{3 \mathrm{r}}\left[\int_{\Gamma^{+}} g_{3}^{+} \mathrm{d} \Gamma+\int_{\Gamma^{-}} g_{3}^{-} \mathrm{d} \Gamma\right]+\frac{H_{3 \mathrm{r}} h}{L} \int_{\Gamma_{0}} H_{3} \mathrm{~d} s \mathrm{~d} x_{3}=0 .
\end{aligned}
$$

Si l'on souhaite donc que les efforts $f_{3}, g_{3}$ d'une part et $f_{\mathrm{t}}, g_{\mathrm{t}}$ d'autre part interviennent au même ordre dans le développement asymptotique des équations, on doit avoir :

$$
g_{\mathrm{tr}}=h f_{\mathrm{tr}} \quad g_{3 \mathrm{r}}=h f_{3 \mathrm{r}}
$$

ou de façon équivalente :

$$
\mathcal{F}_{\mathrm{t}}=\mathcal{G}_{\mathrm{t}} \quad \text { et } \quad \mathcal{F}_{3}=\mathcal{G}_{3} .
$$

Nous supposerons d'autre part que la pesanteur est le seul effort volumique qui intervient dans les équations. À titre d'exemple, dans le cas d'une plaque (en acier de construction, en plomb ou en graphite), d'épaisseur $10^{-2} \mathrm{~m}$, de diamètre $1 \mathrm{~m}$ et soumise à son poids, $\mathcal{F}_{3}$ est de l'ordre de $10^{-8}=\varepsilon^{4}$ et $\mathcal{F}_{\mathbf{t}}=0$ (la composante tangentielle du poids est nulle). Il est donc physiquement raisonnable de considérer des niveaux d'efforts pour lesquels $\mathcal{F}_{3}=\mathcal{G}_{3}=\varepsilon^{4}$ et $\mathcal{F}_{\mathrm{t}}=0$. Nous considérerons enfin des efforts tangentiels surfaciques beaucoup plus importants $\left(\mathcal{G}_{\mathrm{t}}=\varepsilon^{3}\right)$ afin d'obtenir des effets membranaires non négligeables. La plupart des cas usuels relèvent de ces hypothèses.

\section{Développement asymptotique des équations}

Étant maintenant ramené à un problème mono-échelle, la mise en cuvre de la technique des développements asymptotiques conduit à postuler l'existence d'un développement en série formelle de la solution $\left(V, u_{3}\right)$.

$$
\begin{aligned}
& V=V^{0}+\varepsilon V^{1}+\varepsilon^{2} V^{2}+\cdots \\
& u_{3}=u_{3}^{0}+\varepsilon u_{3}^{1}+\varepsilon^{2} u_{3}^{2}+\cdots
\end{aligned}
$$


On a alors le résultat suivant :

Proposition 1 Pour des niveaux d'efforts extérieurs donnés vérifiant $\mathcal{F}_{3}=\mathcal{G}_{3}=\varepsilon^{4}$ et $\mathcal{G}_{\mathrm{t}}=\varepsilon^{3}$, on a $V^{0}=0$.

En effet, un calcul similaire à celui effectué plus loin à la proposition 2, montre que pour ces niveaux d'efforts $V^{0}$ ne dépend que de $\left(x_{1}, x_{2}\right)$ et vérifie le problème aux limites :

$$
\left\{\begin{array}{l}
\operatorname{div} n_{\mathrm{t}}^{0}=0 \text { dans } \omega \\
\left.V^{0}\right|_{\partial \omega}=0
\end{array}\right.
$$

où :

$$
n_{\mathrm{t}}^{0}=\frac{4 \beta}{2+\beta} \operatorname{Tr} e_{\mathrm{t}}\left(V^{0}\right) I_{2}+4 e_{\mathrm{t}}\left(V^{0}\right) \quad e_{\mathrm{t}}\left(V^{0}\right)=\frac{1}{2}\left(\operatorname{grad} V^{0}+\overline{\operatorname{grad} V^{0}}\right) .
$$

Il est bien connu (moyennant certaines hypothèses de régularité ) que ce problème aux lımites admet une unique solution dans $H_{0}^{1}(\omega): V^{0}=0$.

On peut alors écrire

$$
V=\frac{V^{*}}{V_{\mathrm{r}}}=\frac{V^{*}}{h}=\varepsilon V^{1}+\varepsilon^{2} V^{2}+\cdots
$$

soit de façon équivalente :

$$
\widetilde{V}=\frac{V^{*}}{\varepsilon V_{\mathrm{r}}}=\frac{V^{*}}{\varepsilon h}=V^{1}+\varepsilon V^{2}+\cdots=\widetilde{V^{0}}+\varepsilon \widetilde{V^{1}}+\varepsilon^{2} \widetilde{V^{2}}+\cdots
$$

On voit donc que pour ces niveaux d'efforts l'échelle des déplacements tangentiels $V_{\mathrm{r}}=u_{3 \mathrm{r}}=h$ est mal choisie, et qu'il faut prendre $V_{\mathrm{r}}=\varepsilon u_{3 \mathbf{r}}=\varepsilon h$ afin que $\widetilde{V}$ soit de l'ordre de l'unité. Il convient donc de "réadimensionnaliser" le déplacement tangentiel . $V^{*}$ avec $V_{\mathrm{r}}=\varepsilon u_{3 \mathrm{r}}$ comme échelle de référence.

La relation $V_{\mathrm{r}}=\varepsilon u_{3 \mathrm{r}}$, qui est obtenue ici comme conséquence de notre démarche, est formellement équivalente aux hypothèses sur les changements d'échelle effectuées dans [4-6, 15] aussi bien dans le cas linéaire que dans le cas non linéaire Elle traduit le fait que le déplacement tangentiel $V^{*}$ est très inférieur à la déflexion $u_{3}^{*}$ et conduit (proposition 2 ) aux hypothèses de Kirchhoff-Love. Considérer $V_{\mathrm{r}}=\varepsilon u_{3 \mathrm{r}}$ comme hypothèse a prori revient à avoir déjà une idée intuitive de la solution.

Nous effectuons donc une nouvelle adimensionnalisation des équations d'équilıbre avec cette fois $u_{3 \mathrm{r}}=h, V_{\mathrm{r}}=\varepsilon h$ et nous continuerons à noter les composantes du déplacement adimensionnel par $V$ et $u_{3}$.

Proposition 2: Pour des niveaux d'efforts extérieurs donnés vérifiant $\mathcal{F}_{3}=\mathcal{G}_{3}=\varepsilon^{4}$ et $\mathcal{G}_{t}=\varepsilon^{3}$, le premier terme $\left(V^{0}, u_{3}^{0}\right)$ du développement de $\left(V, u_{3}\right)$ est un déplacement de Kirchhoff-Love qui satisfait à :

i) $u_{3}^{0}=\zeta_{3}^{0}\left(x_{1}, x_{2}\right) \quad V^{0}=\zeta_{\mathrm{t}}^{0}\left(x_{1}, x_{2}\right)-x_{3} \operatorname{grad} \zeta_{3}^{0}$

ii) $\zeta_{3}^{0}$ est solution du problème de flexion

$$
\frac{8}{3} \frac{\beta+1}{\beta+2} \Delta^{2} \zeta_{3}^{0}=p_{3}+\operatorname{div} M_{\mathrm{t}} \quad \text { avec }\left.\quad u_{3}^{0}\right|_{\partial \omega}=\left.\frac{\partial u_{3}^{0}}{\partial n}\right|_{\partial \omega}=0
$$

où l'on a posé :

$$
M_{\mathrm{t}}=g_{\mathrm{t}}^{+}-g_{\mathrm{t}}^{-} \quad p_{3}=\int_{-1}^{1} f_{3} \mathrm{~d} x_{3}+\left(g_{3}^{+}+g_{3}^{-}\right)
$$


iii) $\zeta_{\mathrm{t}}^{0}$ est solution du problème de membrane

$$
\begin{gathered}
\operatorname{div} n_{\mathrm{t}}^{0}=-\left.p_{\mathrm{t}} \quad \operatorname{avec} \quad \zeta_{\mathrm{t}}^{0}\right|_{\partial \omega}=0 \\
\text { où : } \quad n_{\mathrm{t}}^{0}=\frac{4 \beta}{2+\beta} \operatorname{Tr} e_{\mathrm{t}}\left(\zeta_{\mathrm{t}}^{0}\right) I_{2}+4 e_{\mathrm{t}}\left(\zeta_{\mathrm{t}}^{0}\right) \quad e_{\mathrm{t}}\left(\zeta_{\mathrm{t}}^{0}\right)=\frac{1}{2}\left(\operatorname{grad} \zeta_{\mathrm{t}}^{0}+\overline{\operatorname{grad} \zeta_{\mathrm{t}}^{0}}\right) \\
p_{\mathrm{t}}=g_{\mathrm{t}}^{+}+g_{\mathrm{t}}^{-}
\end{gathered}
$$

Démonstratıon : La démonstration de ce résultat comporte trois étapes numérotées de i) à iii). 2) $\left(u_{3}^{0}, V^{0}\right)$ est un déplacement de Kirchhoff-Love.

Pour des niveaux d'efforts de type $\mathcal{F}_{3}=\mathcal{G}_{3}=\varepsilon^{4}, \mathcal{F}_{\mathrm{t}}=0$ et $\mathcal{G}_{\mathrm{t}}=\varepsilon^{3}$, l'adimensionnalisation des équations d'équilibre (1)-(2) avec $u_{3 \mathrm{r}}=h$ et $V_{\mathrm{r}}=\varepsilon u_{3 \mathrm{r}}$, conduit sur $\left.\Omega=\omega \times\right]-1,1$ [à :

$$
\begin{array}{ll}
\varepsilon^{2}[(1+\beta) \operatorname{grad} \operatorname{div} V+\Delta V]+(1+\beta) \operatorname{grad} \frac{\partial u_{3}}{\partial x_{3}}+\frac{\partial^{2} V}{\partial x_{3}^{2}} & =0 \\
\varepsilon^{2}\left[(1+\beta) \frac{\partial}{\partial x_{3}} \operatorname{div} V+\Delta u_{3}\right]+(2+\beta) \frac{\partial^{2} u_{3}}{\partial x_{3}^{2}} & =-\varepsilon^{4} f_{3} .
\end{array}
$$

Les conditıons aux limites sur les faces supérieure et inférieure sont données par

$$
\begin{array}{lll}
\operatorname{grad} u_{3}+\frac{\partial V}{\partial x_{3}} & = \pm \varepsilon^{2} g_{\mathrm{t}}^{ \pm} \quad \text { pour } x_{3}= \pm 1 \\
(2+\beta) \frac{\partial u_{3}}{\partial x_{3}}+\varepsilon^{2} \beta \operatorname{div} V & = \pm \varepsilon^{4} g_{3}^{ \pm} & \text {pour } x_{3}= \pm 1
\end{array}
$$

On postule à nouveau l'exıstence d'un développement en sérıe formelle de la solution "réadimensionnalisée" $\left(V, u_{3}\right)$ :

$$
\begin{gathered}
V=V^{0}+\varepsilon V^{1}+\varepsilon^{2} V^{2}+\cdots \\
u_{3}=u_{3}^{0}+\varepsilon u_{3}^{1}+\varepsilon^{2} u_{3}^{2}+\cdots
\end{gathered}
$$

En reportant les développements de $V$ et de $u_{3}$ dans les équations (6) et (7) et en annulant successivement les facteurs des différentes puissances de $\varepsilon$, on obtient des problèmes couplés $\mathcal{P}_{0}, \mathcal{P}_{1}, \mathcal{P}_{2} \ldots$ faisant intervenir les inconnues du développement (8). Le problème $\mathcal{P}_{0}$ s'écrit alors :

$$
\begin{gathered}
(1+\beta) \operatorname{grad} \frac{\partial u_{3}^{0}}{\partial x_{3}}+\frac{\partial^{2} V^{0}}{\partial x_{3}^{2}}=0 \text { dans } \Omega, \\
(2+\beta) \frac{\partial^{2} u_{3}^{0}}{\partial x_{3}^{2}}=0 \quad \text { dans } \Omega \\
\frac{\partial V^{0}}{\partial x_{3}}=-\operatorname{grad} u_{3}^{0} \quad \text { pour } x_{3}= \pm 1 \\
(2+\beta) \frac{\partial u_{3}^{0}}{\partial x_{3}}=0 \quad \text { pour } x_{3}= \pm 1 .
\end{gathered}
$$

L'équation (10) nous donne $u_{3}^{0}=x_{3} \phi_{3}^{0}\left(x_{\alpha}\right)+\zeta_{3}^{0}\left(x_{\alpha}\right)$ et $(12)$ implique alors $\phi_{3}^{0}=0$. On a donc :

$$
u_{3}^{0}=\zeta_{3}^{0}\left(x_{1}, x_{2}\right)
$$


D'autre part, (9) et (13) impliquent :

$$
V^{0}=-x_{3} \phi\left(x_{\alpha}\right)+\zeta_{\mathrm{t}}^{0}\left(x_{1}, x_{2}\right)
$$

qui devient avec (11) :

$$
V^{0}=\zeta_{t}^{0}\left(x_{1}, x_{2}\right)-x_{3} \operatorname{grad} u_{3}^{0} .
$$

Le point i) de la proposition 2 est ainsi démontré.

Les problèmes étant couplés deux à deux de façon décalée $\left(\mathcal{P}_{0}\right.$ est couplé avec $\mathcal{P}_{2}, \mathcal{P}_{4} \ldots$ alors que $\mathcal{P}_{1}$ est couplé avec $\left.\mathcal{P}_{3}, \mathcal{P}_{5} \ldots\right)$, le problème $\mathcal{P}_{1}$ conduit à un résultat similaire pour $u_{3}^{1}$ et $V^{1}$. Même si ce résultat ne sert pas dans la démonstration des points ii) et iii), il sera nécessaire au calcul des contraintes et se trouve donc explicité ici.

Les équations du problème $\mathcal{P}_{1}$ sont similaires à celles du problème $\mathcal{P}_{0}$ :

$$
\begin{gathered}
(1+\beta) \operatorname{grad} \frac{\partial u_{3}^{1}}{\partial x_{3}}+\frac{\partial^{2} V^{1}}{\partial x_{3}^{2}}=0 \quad \text { dans } \Omega \\
(2+\beta) \frac{\partial^{2} u_{3}^{1}}{\partial x_{3}^{2}}=0 \quad \text { dans } \Omega \\
\frac{\partial V^{1}}{\partial x_{3}}=-\operatorname{grad} u_{3}^{1} \quad \text { pour } x_{3}= \pm 1 \\
(2+\beta) \frac{\partial u_{3}^{1}}{\partial x_{3}}=0 \quad \text { pour } x_{3}= \pm 1
\end{gathered}
$$

Ainsi on a :

$$
\begin{aligned}
& u_{3}^{1}=\zeta_{3}^{1}\left(x_{1}, x_{2}\right) \\
& V^{1}=\zeta_{\mathrm{t}}^{1}\left(x_{1}, x_{2}\right)-x_{3} \operatorname{grad} u_{3}^{1} .
\end{aligned}
$$

ıv) Équation de flexion.

L'annulation du facteur de $\varepsilon^{2}$ conduit au problème $\mathcal{P}_{2}$ :

$$
\begin{gathered}
(1+\beta) \operatorname{grad} \operatorname{div} V^{0}+\Delta V^{0}+(1+\beta) \operatorname{grad} \frac{\partial u_{3}^{2}}{\partial x_{3}}+\frac{\partial^{2} V^{2}}{\partial x_{3}^{2}}=0 \quad \text { dans } \Omega, \\
(1+\beta) \frac{\partial}{\partial x_{3}} \operatorname{div} V^{0}+\Delta u_{3}^{0}+(2+\beta) \frac{\partial^{2} u_{3}^{2}}{\partial x_{3}^{2}}=0 \quad \text { dans } \Omega, \\
\operatorname{grad} u_{3}^{2}+\frac{\partial V^{2}}{\partial x_{3}}= \pm g_{\mathrm{t}}^{ \pm} \quad \text { pour } x_{3}= \pm 1, \\
(2+\beta) \frac{\partial u_{3}^{2}}{\partial x_{3}}+\beta \operatorname{div} V^{0}=0 \quad \text { pour } x_{3}= \pm 1 .
\end{gathered}
$$

En remplaçant alors $V^{0}$ par son expression trouvée en (14), l'équation (20) peut s'écrire sous la forme :

$$
-x_{3}(2+\beta) \operatorname{grad} \Delta u_{3}^{0}+\beta \operatorname{grad} \frac{\partial u_{3}^{2}}{\partial x_{3}}+\frac{\partial}{\partial x_{3}}\left(\operatorname{grad} u_{3}^{2}+\frac{\partial V^{2}}{\partial x_{3}}\right)+\left[(1+\beta) \operatorname{grad} \operatorname{div} \zeta_{\mathrm{t}}^{0}+\Delta \zeta_{\mathrm{t}}^{0}\right]=0 .
$$


En multipliant maintenant la dernière expression par $x_{3}$ et en intégrant par rapport à l'épaisseur après avoir pris la divergence, il vient :

$$
-\frac{2}{3}(2+\beta) \Delta^{2} u_{3}^{0}+\beta \int_{-1}^{1} x_{3} \frac{\partial}{\partial x_{3}} \Delta u_{3}^{2} \mathrm{~d} x_{3}+\int_{-1}^{1} x_{3} \frac{\partial}{\partial x_{3}}\left(\Delta u_{3}^{2}+\frac{\partial}{\partial x_{3}} \operatorname{div} V^{2}\right) \mathrm{d} x_{3}=0 .
$$

Mais, compte tenu de (14), (21) peut s'écrire sous la forme :

$$
-\beta \Delta u_{3}^{0}+(2+\beta) \frac{\partial^{2} u_{3}^{2}}{\partial x_{3}^{2}}=0
$$

qui devient en tenant compte de la condition aux limites (23) :

$$
\frac{\partial u_{3}^{2}}{\partial x_{3}}=-\frac{\beta}{2+\beta}\left(-x_{3} \Delta u_{3}^{0}+\operatorname{div} \zeta_{t}^{0}\right)=-\frac{\beta}{2+\beta} \operatorname{div} V^{0}
$$

Remplaçant alors :

$$
\int_{-1}^{1} x_{3} \frac{\partial}{\partial x_{3}} \Delta u_{3}^{2} \mathrm{~d} x_{3}=\frac{2 \beta}{3(2+\beta)} \Delta^{2} u_{3}^{0}
$$

dans (25), on obtient:

$$
-\frac{8}{3} \frac{\beta+1}{\beta+2} \Delta^{2} u_{3}^{0}+\int_{-1}^{1} x_{3} \frac{\partial}{\partial x_{3}}\left(\Delta u_{3}^{2}+\frac{\partial}{\partial x_{3}} \operatorname{div} V^{2}\right) \mathrm{d} x_{3}=0 .
$$

D'autre part, l'intégration par partie du second terme de (28) conduit à :

$$
\int_{-1}^{1} x_{3} \frac{\partial}{\partial x_{3}}\left(\Delta u_{3}^{2}+\frac{\partial}{\partial x_{3}} \operatorname{div} V^{2}\right) \mathrm{d} x_{3}=\left[x_{3}\left(\Delta u_{3}^{2}+\frac{\partial}{\partial x_{3}} \operatorname{div} V^{2}\right)\right]_{-1}^{1}-\left\{\int_{-1}^{1} \Delta u_{3}^{2} \mathrm{~d} x_{3}+\left[\operatorname{div} V^{2}\right]_{-1}^{1}\right\}
$$

et avec la condition aux limites (22) le premier terme du second membre devient :

$$
\left[x_{3}\left(\Delta u_{3}^{2}+\frac{\partial}{\partial x_{3}} \operatorname{div} V^{2}\right)\right]_{-1}^{1}=\operatorname{div}\left(g_{t}^{+}-g_{t}^{-}\right)
$$

L'équation (28) s'écrit alors :

$$
\frac{8}{3} \frac{\beta+1}{\beta+2} \Delta^{2} u_{3}^{0}+\underbrace{\int_{-1}^{1} \Delta u_{3}^{2} d x_{3}+\left[\operatorname{div} V^{2}\right]_{-1}^{1}}_{(a)}=\operatorname{div} M_{\mathrm{t}}
$$

où l'on a posé :

$$
M_{\mathrm{t}}=g_{\mathrm{t}}^{+}-g_{\mathrm{t}}^{-} \text {. }
$$

Il convient maintenant d'expliciter le problème $\mathcal{P}_{4}$ pour éliminer le terme $(a)$.

La deuxième équation et la deuxième condition aux limites du problème $\mathcal{P}_{\mathbf{4}}$ s'écrivent :

$$
\begin{gathered}
(1+\beta) \frac{\partial}{\partial x_{3}} \operatorname{div} V^{2}+\Delta u_{3}^{2}+(2+\beta) \frac{\partial^{2} u_{3}^{4}}{\partial x_{3}^{2}}=-f_{3} \quad \text { dans } \Omega \\
(2+\beta) \frac{\partial u_{3}^{4}}{\partial x_{3}}+\beta \operatorname{div} V^{2}= \pm g_{3}^{ \pm} \quad \text { pour } x_{3}= \pm 1
\end{gathered}
$$


En intégrant alors (29) entre -1 et 1 , on a .

$$
\left[(1+\beta) \operatorname{div} V^{2}+(2+\beta) \frac{\partial u_{3}^{4}}{\partial x_{3}}\right]_{-1}^{1}+\int_{-1}^{1} \Delta u_{3}^{2} \mathrm{~d} x_{3}=-\int_{-1}^{1} f_{3} \mathrm{~d} x_{3}
$$

qui devient avec $(30)$.

$$
\underbrace{\left[\operatorname{div} V^{2}\right]_{-1}^{1}+\int_{-1}^{1} \Delta u_{3}^{2} \mathrm{~d} x_{3}}_{(a)}=-\left[\int_{-1}^{1} f_{3} \mathrm{~d} x_{3}+\left(g_{3}^{+}+g_{3}^{-}\right)\right] .
$$

On obtient donc finalement :

$$
\frac{8}{3} \frac{\beta+1}{\beta+2} \Delta^{2} u_{3}^{0}=\int_{-1}^{1} f_{3} \mathrm{~d} x_{3}+\left(g_{3}^{+}+g_{3}^{-}\right)+\operatorname{div} M_{\mathrm{t}}
$$

qui constitue après retour au domaine initial (voir paragraphe 7) l'équation standard bidimensionnelle des plaques minces en flexion. Le point ii) de la proposition 2 est ainsi prouvé.

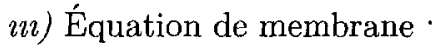

En intégrant maintenant directement (24) sur l'épaisseur. il reste :

$$
\int_{-1}^{1} \beta \operatorname{grad} \frac{\partial u_{3}^{2}}{\partial x_{3}} \mathrm{~d} x_{3}+\left[\operatorname{grad} u_{3}^{2}+\frac{\partial V^{2}}{\partial x_{3}}\right]_{-1}^{1}+2\left[(1+\beta) \operatorname{grad}\left(\operatorname{div} \zeta_{\mathrm{t}}^{0}\right)+\Delta \zeta_{\mathrm{t}}^{0}\right]=0 .
$$

Utilisant alors d'une part la condition aux limites (22)

$$
\left[\operatorname{grad} u_{3}^{2}+\frac{\partial V^{2}}{\partial x_{3}}\right]_{-1}^{1}=g_{\mathrm{t}}^{+}+g_{\mathrm{t}}^{-}
$$

et d'autre part l'équation de couplage (27)

$$
\frac{\partial u_{3}^{2}}{\partial x_{3}}=-\frac{\beta}{2+\beta}\left(-x_{3} \Delta u_{3}^{0}+\operatorname{div} \zeta_{\mathbf{t}}^{0}\right)=-\frac{\beta}{2+\beta} \operatorname{div} V^{0}
$$

l'équation (32) devient :

$$
\frac{4+6 \beta}{2+\beta} \operatorname{grad} \operatorname{div} \zeta_{\mathrm{t}}^{0}+2 \Delta \zeta_{\mathrm{t}}^{0}=-\left(g_{\mathrm{t}}^{+}+g_{\mathrm{t}}^{-}\right)
$$

Il est également possible d'écrire (33) de façon plus intrinsèque sous la forme :

$$
\operatorname{div} n_{\mathrm{t}}^{0}=-p_{\mathrm{t}}
$$

où l'on a posé :

$$
\begin{gathered}
n_{\mathrm{t}}^{0}=\frac{4 \beta}{2+\beta} \operatorname{Tr}\left(e_{\mathrm{t}}\left(\zeta_{\mathrm{t}}^{0}\right)\right) I_{2}+4 e_{\mathrm{t}}\left(\zeta_{\mathrm{t}}^{0}\right) \\
e_{\mathrm{t}}\left(\zeta_{\mathrm{t}}^{0}\right)=\frac{1}{2}\left(\operatorname{grad} \zeta_{\mathrm{t}}^{0}+\overline{\operatorname{grad} \zeta_{\mathrm{t}}^{0}}\right) \quad \text { et } \quad p_{\mathrm{t}}=g_{\mathrm{t}}^{+}+g_{\mathrm{t}}^{-}
\end{gathered}
$$

La proposition 2 est ainsi démontrée. 
Remarque - On aurait pu également adimensionnaliser directement le déplacement $U^{*}$ avec $V_{\mathrm{r}}=u_{3 \mathrm{r}}=L$. Compte tenu de la linéarité des équations, cela revient à diviser par $\varepsilon$ les premiers membres des équations adimensionnelles (3) et (4). En mettant en oeuvre la technique des développements asymptotiques, on obtiendrait les mêmes équations qu'à la proposition 2 avec un second membre nul, équations qui admettent comme unique solution $\left(V_{0}, u_{3}^{0}\right)=(0,0)$ dans $\left[H_{0}^{1}(\omega)\right]^{2} \times H_{0}^{2}(\omega)$. On serait alors conduit à "réadimensionnaliser" le déplacement avec $V_{\mathrm{r}}=u_{3 \mathrm{r}}=h$, ce qui nous ramène au cas traité ici

\section{Calcul des contraintes}

Il est maintenant possible de déduire, de la loi de comportement tridimensionnelle ainsi que des résultats précédemment obtenus, l'expression des composantes du tenseur des contraintes. Dans le cadre de l'élasticité linéaire, la loi de comportement s'écrit :

$$
\sigma^{*}=\lambda \operatorname{Tr}\left(e^{*}\right) I+2 \mu e^{*}
$$

où :

$$
e^{*}=\frac{1}{2}\left(\operatorname{Grad}^{*} U^{*}+\overline{\operatorname{Grad}^{*} U^{*}}\right)
$$

représente le tenseur linéarisé des déformations.

Décomposant $U^{*}$ sous la forme $U^{*}=V^{*}+u_{3}^{*} e_{3}$, les composantes du tenseur des contraintes s'écrivent alors .

$$
\sigma^{*}=\left(\begin{array}{cc}
\lambda\left[\operatorname{div}^{*} V^{*}+\frac{\partial u_{3} *}{\partial x_{3}^{*}}\right] I_{2}+2 \mu e_{t}^{*}\left(V^{*}\right) & \mu\left[\frac{\partial V^{*}}{\partial x_{3}^{*}}+\operatorname{grad}^{*} u_{3}^{*}\right] \\
\mu\left[\overline{\left.\frac{\partial V^{*}}{\partial x_{3}^{*}}+\overline{\operatorname{grad}^{*} u_{3}^{*}}\right]}\right. & \lambda \operatorname{div}^{*} V^{*}+(\lambda+2 \mu) \frac{\partial u_{3}^{*}}{\partial x_{3}^{*}}
\end{array}\right)=\left(\begin{array}{cc}
\sigma_{\mathrm{t}}^{*} & \sigma_{s}^{*} \\
\overline{\sigma_{s}^{*}} & \sigma_{n}^{*}
\end{array}\right)
$$

où l'on a posé :

$$
e_{\mathrm{t}}^{*}\left(V^{*}\right)=\frac{1}{2}\left(\operatorname{grad}^{*} V^{*}+\overline{\operatorname{grad}^{*} V^{*}}\right)
$$

et où $I_{2}$ désigne l'identité de $\mathbb{R}^{2}$.

Adimensionnalisant alors $\sigma^{*}$ composante par composante avec $u_{3 \mathrm{r}}=h$ et $V_{\mathrm{r}}=\varepsilon u_{3 \mathrm{r}}$, on obtient :

$$
\begin{aligned}
\frac{\sigma_{\mathrm{t}}^{*}}{\mu} & =\beta \frac{\partial u_{3}}{\partial x_{3}} I_{2}+\varepsilon^{2}\left[\beta \operatorname{div} V I_{2}+2 e_{\mathrm{t}}(V)\right] \\
\frac{\sigma_{s}^{*}}{\mu} & =\varepsilon\left[\operatorname{grad} u_{3}+\frac{\partial V}{\partial x_{3}}\right] \\
\frac{\sigma_{n}^{*}}{\mu} & =(2+\beta) \frac{\partial u_{3}}{\partial x_{3}}+\varepsilon^{2} \beta \operatorname{div} V
\end{aligned}
$$

où :

$$
e_{\mathrm{t}}(V)=\frac{1}{2}(\operatorname{grad} V+\overline{\operatorname{grad} V})
$$

Remplaçons maintenant $V$ et $u_{3}$ par leurs développements (8) et utilisons certains résultats 
précédemment obtenus pour simplifier les expressions. D'une part nous avons les hypothèses cinématiques de Kirchhoff-love vérifiées par les deux premiers termes du développement de $\left(V, u_{3}\right)$ qui s'écrivent :

$$
\frac{\partial u_{3}^{0}}{\partial x_{3}}=0 \quad \frac{\partial V^{0}}{\partial x_{3}}+\operatorname{grad} u_{3}^{0}=0 \quad \text { et } \quad \frac{\partial u_{3}^{1}}{\partial x_{3}}=0 \quad \frac{\partial V^{1}}{\partial x_{3}}+\operatorname{grad} u_{3}^{1}=0
$$

D'autre part, le problème $\mathcal{P}_{3}$ conduit de façon analogue à une autre relation de couplage, similaire à (27), où les indices sont simplement décalés :

$$
\frac{\partial u_{3}^{3}}{\partial x_{3}}=-\frac{\beta}{2+\beta} \operatorname{div} V^{1}
$$

Utilisant alors les relations de couplage (27) et (36), ainsi que les hypothèses cinématiques de Kirchhoff-love ci-dessus, (35) peut s'écrire sous la forme :

$$
\begin{aligned}
\frac{\sigma_{\mathrm{t}}^{*}}{\varepsilon^{2} \mu} & =\frac{2 \beta}{2+\beta} \operatorname{div} V^{0} I_{2}+2 e_{\mathrm{t}}\left(V^{0}\right)+\varepsilon\left[\frac{2 \beta}{2+\beta} \operatorname{div} V^{1} I_{2}+2 e_{\mathrm{t}}\left(V^{1}\right)\right]+O\left(\varepsilon^{2}\right) \\
\frac{\sigma_{s}^{*}}{\varepsilon^{2} \mu} & =\varepsilon\left[\operatorname{grad} u_{3}^{2}+\frac{\partial V^{2}}{\partial x_{3}}\right]+O\left(\varepsilon^{2}\right) \\
\frac{\sigma_{n}^{*}}{\varepsilon^{2} \mu} & =\varepsilon^{2}\left[(2+\beta) \frac{\partial u_{3}^{4}}{\partial x_{3}}+\beta \operatorname{div} V^{2}\right]+O\left(\varepsilon^{3}\right) .
\end{aligned}
$$

Le choix de l'échelle de référence des contraintes $\sigma_{\mathrm{r}}=\varepsilon^{2} \mu$ apparaît alors naturellement. En effet, si on choisit la même contrainte de référence pour toutes les composantes de $\sigma^{*}$, l'adimensionnalisation des contraintes doit être telle qu'au moins une composante de $\sigma=\frac{\sigma^{*}}{\sigma_{\mathrm{r}}}$ solt de l'ordre de 1.

L'existence d'un développement de la solution $\left(V, u_{3}\right)$ en puissances de $\varepsilon$ implique donc l'existence d'un développement de $\sigma$ en puissances de $\varepsilon$ :

$$
\sigma=\sigma^{0}+\varepsilon \sigma^{1}+\varepsilon^{2} \sigma^{2}+\cdots
$$

avec :

$$
\begin{array}{ll}
\sigma_{\mathrm{t}}^{0}=\frac{2 \beta}{2+\beta} \operatorname{Tr} e_{\mathrm{t}}\left(V^{0}\right) I_{2}+2 e_{\mathrm{t}}\left(V^{0}\right) & \\
\sigma_{\mathrm{s}}^{1}=\operatorname{grad} u_{3}^{2}+\frac{\partial V^{2}}{\partial x_{3}} & \sigma_{\mathrm{s}}^{0}=0 \\
\sigma_{\mathrm{n}}^{2}=(2+\beta) \frac{\partial u_{3}^{4}}{\partial x_{3}}+\beta \operatorname{div} V^{2} & \sigma_{\mathrm{n}}^{1}=0 \quad \sigma_{\mathrm{n}}^{0}=0
\end{array}
$$

Il est également possible d'écrire $\sigma_{\mathrm{t}}^{0}$ sous la forme

$$
\sigma_{\mathrm{t}}^{0}=\frac{1}{2} n_{\mathrm{t}}^{0}+\frac{3}{2} x_{3} m_{\mathrm{t}}^{0}
$$


où l'on a posé :

$$
\begin{gathered}
m_{\mathrm{t}}^{0}=-\left\{\frac{4 \beta}{3(2+\beta)} \Delta \zeta_{3}^{0} I_{2}+\frac{4}{3} \operatorname{grad}\left(\operatorname{grad} \zeta_{3}^{0}\right)\right\} \\
n_{\mathrm{t}}^{0}=\frac{4 \beta}{2+\beta} \operatorname{Tr} e_{\mathrm{t}}\left(\zeta_{\mathrm{t}}^{0}\right) I_{2}+4 e_{\mathrm{t}}\left(\zeta_{\mathrm{t}}^{0}\right) \quad \text { et } \quad e_{\mathrm{t}}\left(\zeta_{\mathrm{t}}^{0}\right)=\frac{1}{2}\left(\operatorname{grad} \zeta_{\mathrm{t}}^{0}+\overline{\operatorname{grad} \zeta_{\mathrm{t}}^{0}}\right) .
\end{gathered}
$$

Les expressions explicites de $\sigma_{\mathrm{s}}^{1}$ et de $\sigma_{\mathrm{n}}^{2}$ s'obtiennent maintenant par intégration sur l'épaisseur. La démarche est analogue à celle développée par P.G. Ciarlet et P. Destuynder dans [4] et [6]. Calcul $\sigma_{\mathrm{s}}^{1}$ :

Prenant le gradient de (27) et rempaçant l'expression ainsi obtenue dans (20), il vient :

$$
\frac{2+3 \beta}{2+\beta} \operatorname{grad} \operatorname{div} V^{0}+\Delta V^{0}+\frac{\partial^{2} V^{2}}{\partial x_{3}^{2}}+\operatorname{grad} \frac{\partial u_{3}^{2}}{\partial x_{3}}=0 .
$$

Compte tenu alors de (38) et des conditions aux limites sur les faces supérieure et inférieure, on a :

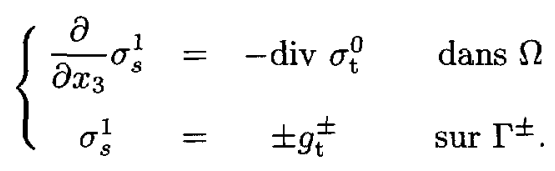

L'intégration de l'équation précédente conduit à :

$$
\sigma_{s}^{1}=-\frac{2(1+\beta)}{2+\beta}\left(1-x_{3}^{2}\right) \operatorname{grad} \Delta u_{3}^{0}+\frac{g_{\mathrm{t}}^{+}-g_{\mathrm{t}}^{-}}{2}+\frac{x_{3}}{2}\left(g_{\mathrm{t}}^{+}+g_{\mathrm{t}}^{-}\right)
$$

où l'équation d'équilibre (31) a été utilisée afin de regrouper certains termes.

Calcul de $\sigma_{\mathrm{n}}^{2}$ :

L'égalité (29) s'écrit encore:

$$
\beta \frac{\partial \operatorname{div} V^{2}}{\partial x_{3}}+(2+\beta) \frac{\partial^{2} u_{3}^{4}}{\partial x_{3}^{2}}+\Delta u_{3}^{2}+\frac{\partial \operatorname{div} V^{2}}{\partial x_{3}}=-f_{3}
$$

On obtient alors compte tenu de (38):

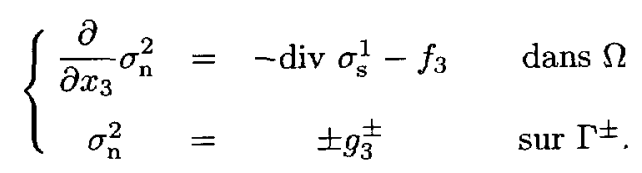

Compte tenu de (40), l'intégration de $\sigma_{\mathrm{n}}^{2}$ conduit à :

$$
\begin{aligned}
\sigma_{\mathrm{n}}^{2} & =\frac{2 x_{3}}{3}\left(1-x_{3}^{2}\right) \frac{\beta+1}{\beta+2} \Delta^{2} u_{3}^{0}+\frac{1+x_{3}}{2} \int_{-1}^{1} f_{3} \mathrm{~d} x_{3}-\int_{-1}^{x_{3}} f_{3}(t) \mathrm{d} t \\
& +\frac{g_{3}^{+}-g_{3}^{-}}{2}+\frac{x_{3}}{2}\left(g_{3}^{+}+g_{3}^{-}\right)+\frac{1}{4}\left(1-x_{3}^{2}\right) \operatorname{div}\left(g_{\mathrm{t}}^{+}+g_{\mathrm{t}}^{-}\right)
\end{aligned}
$$




\section{Retour aux variables initiales}

Le retour au domaine initial $\Omega^{*}$ ainsi qu'aux variables physıques $V^{*}, u_{3}^{*}, f^{*}$ et $g^{*}$ conduit à définir :

$$
\begin{aligned}
& V^{* 0}=V_{\mathrm{r}} V^{0}=\varepsilon h V^{0} \\
& u_{3}^{* 0}=u_{3 \mathrm{r}} u_{3}^{0}=h u_{3}^{0}
\end{aligned}
$$

On a alors le résultat suivant .

Proposition 3 : Le déplacement $\left(V^{* 0}, u_{3}^{* 0}\right)$ est un déplacement de Kirchhoff-Love qui vérifie l'équation standard bibimensionnelle des plaques minces en flexion :

$$
\begin{aligned}
u_{3}^{* 0}=\zeta_{3}^{* 0}\left(x_{1}^{*}, x_{2}^{*}\right) \quad V^{* 0} & =\zeta_{\mathrm{t}}^{* 0}\left(x_{1}^{*}, x_{2}^{*}\right)-x_{3}^{*} \operatorname{grad} \zeta_{3}^{* 0} \\
\frac{2}{3} \frac{E h^{3}}{1-\nu^{2}} \Delta^{* 2} u_{3}^{* 0} & =p_{3}^{*}+\operatorname{div}^{*} M_{\mathrm{t}}^{*} \\
h \operatorname{div}^{*} n_{\mathrm{t}}^{* 0} & =-p_{\mathrm{t}}^{*}
\end{aligned}
$$

où :

$$
\begin{array}{cc}
n_{\mathrm{t}}^{* 0}=\frac{4 \lambda \mu}{\lambda+2 \mu} \operatorname{Tr} e_{\mathrm{t}}^{*}\left(\zeta^{* 0}\right) I_{2}+4 \mu e_{\mathrm{t}}^{*}\left(\zeta^{* 0}\right) & e_{\mathrm{t}}^{*}\left(\zeta^{* 0}\right)=\frac{1}{2}\left(\operatorname{grad}^{*} \zeta_{\mathrm{t}}^{* 0}+\overline{\operatorname{grad}^{*} \zeta_{\mathrm{t}}^{* 0}}\right) \\
M_{\mathrm{t}}^{*}=h\left(g_{\mathrm{t}}^{*+}-g_{\mathrm{t}}^{*-}\right), \quad p_{\mathrm{t}}^{*}=g_{\mathrm{t}}^{*+}+g_{\mathrm{t}}^{*-}, & p_{3}^{*}=\int_{-h}^{h} f_{3}^{*} \mathrm{~d} x_{3}^{*}+\left(g_{3}^{*+}+g_{3}^{*-}\right) .
\end{array}
$$

En effet, l'équation (31) devient après retour aux variables initiales :

$$
\frac{8}{3} \frac{L^{4} g_{3 \mathrm{r}}}{u_{3 \mathrm{r}}} \frac{\lambda+\mu}{\lambda+2 \mu} \Delta^{* 2} u_{3}^{* 0}=\int_{-h}^{h} f_{3}^{*} \mathrm{~d} x_{3}^{*}+\left(g_{3}^{*+}+g_{3}^{*-}\right)+\frac{L g_{3 \mathrm{r}}}{g_{\mathrm{tr}}} \operatorname{div}^{*}\left(g_{\mathrm{t}}^{*+}-g_{\mathrm{t}}^{*-}\right)
$$

avec $g_{3 \mathbf{r}}=h f_{3 \mathbf{r}}$.

Or compte tenu du choix des niveaux d'efforts, on a :

$$
\frac{L^{4} g_{3 \mathrm{r}}}{u_{3 \mathrm{r}}}=\frac{\mathcal{G}_{3}}{\varepsilon^{4}} \mu h^{3}=\mu h^{3} \quad \text { et } \quad \frac{L g_{3 \mathrm{r}}}{g_{\mathrm{tr}}}={ }^{\prime} h .
$$

Remarquant d'autre part que :

$$
4 \frac{\lambda+\mu}{\lambda+2 \mu} \mu=\frac{E}{1-\nu^{2}}
$$

on obtient finalement :

$$
\frac{2}{3} \frac{E h^{3}}{1-\nu^{2}} \Delta^{* 2} u_{3}^{* 0}=p_{3}^{*}+\operatorname{div}^{*} M_{\mathrm{t}}^{*}
$$

où :

$$
M_{\mathrm{t}}^{*}=h\left(g_{\mathrm{t}}^{*+}-g_{\mathrm{t}}^{*-}\right)
$$

représente le moment de flexion induit par les efforts surfaciques tangentiels. On retrouve bien après retour au domaine initial l'équation standard bidimensionnelle des plaques minces en flexion. 
D'autre part, le retour aux variables dimensionnelles dans l'équation (33) conduit à :

$$
\frac{L^{2} g_{\mathrm{tr}}}{V_{\mathrm{r}}}\left[\frac{4+6 \beta}{2+\beta} \operatorname{grad}^{*}\left(\operatorname{div}^{*} \zeta_{\mathrm{t}}^{* 0}\right)+2 \Delta^{*} \zeta_{\mathrm{t}}^{* 0}\right]=-\left(g_{\mathrm{t}}^{*+}+g_{\mathrm{t}}^{*-}\right)
$$

Or compte tenu des niveaux d'efforts appliqués on a :

$$
\frac{L^{2} g_{\mathrm{tr}}}{V_{\mathrm{r}}}=h \mu \frac{\mathcal{G}_{\mathrm{t}}}{\varepsilon^{3}}=h \mu
$$

d'où l'équation de membrane dimensionnelle énoncée à la propositıon 3 .

Compte tenu maintenant de l'adimensionnalisatıon des contraintes, le retour aux variables physiques conduit à définir :

$$
\sigma_{\mathrm{t}}^{* 0}=\varepsilon^{2} \mu \sigma_{\mathrm{t}}^{0} \quad \sigma_{\mathrm{s}}^{* 0}=\varepsilon^{3} \mu \sigma_{\mathrm{s}}^{1} \quad \sigma_{\mathrm{n}}^{* 0}=\varepsilon^{4} \mu \sigma_{\mathrm{n}}^{2}
$$

On a alors l'expression suivante des contraintes physiques sur l'épaisseur :

Proposition 4 :

$$
\begin{aligned}
\sigma_{\mathrm{t}}^{* 0} & =n_{\mathrm{t}}^{* 0}+\frac{3 x_{3}^{*}}{2} m_{\mathrm{t}}^{* 0} \\
\sigma_{\mathrm{s}}^{* 0} & =-2\left(h^{2}-\left(x_{3}^{*}\right)^{2}\right) \frac{\mu(\lambda+\mu)}{\lambda+2 \mu} \operatorname{grad}^{*} \Delta^{*} \zeta_{3}^{* 0}+\frac{g_{\mathrm{t}}^{*+}-g_{\mathrm{t}}^{*-}}{2}+\frac{x_{3}^{*}}{2 h}\left(g_{\mathrm{t}}^{*+}+g_{\mathrm{t}}^{*-}\right) \\
\sigma_{\mathrm{n}}^{* 0} & =\frac{2}{3} x_{3}^{*}\left(h^{2}-\left(x_{3}^{*}\right)^{2}\right) \frac{\mu(\lambda+\mu)}{\lambda+2 \mu} \Delta^{* 2} \zeta_{3}^{* 0}+\left(\frac{1}{2}+\frac{x_{3}^{*}}{2 h}\right) \int_{-h}^{h} f_{3}^{*} \mathrm{~d} x_{3}^{*}-\int_{-h}^{x_{3}^{*}} f_{3}^{*}\left(t^{*}\right) \mathrm{d} t^{*} \\
& +\frac{1}{2}\left(g_{3}^{*+}-g_{3}^{*-}\right)+\frac{x_{3}^{*}}{2 h}\left(g_{3}^{*+}+g_{3}^{*-}\right)+\frac{1}{4}\left(1-\left(\frac{x_{3}^{*}}{h}\right)^{2}\right) \operatorname{div}^{*}\left[h\left(g_{\mathrm{t}}^{*+}+g_{\mathrm{t}}^{*-}\right)\right]
\end{aligned}
$$

où l'on a posé :

$$
\begin{aligned}
& m_{\mathrm{t}}^{* 0}=-\left\{\frac{4 \lambda \mu}{3(\lambda+2 \mu)} \Delta^{*} \zeta_{3}^{* 0} I_{2}+\frac{4 \mu}{3} \operatorname{grad}^{*}\left(\operatorname{grad}^{*} \zeta_{3}^{* 0}\right)\right\} \\
& n_{\mathrm{t}}^{* 0}=\frac{4 \lambda \mu}{\lambda+2 \mu} \operatorname{Tr} e_{\mathrm{t}}^{*}\left(\zeta^{* 0}\right) I_{2}+4 \mu e_{\mathrm{t}}^{*}\left(\zeta^{* 0}\right)
\end{aligned}
$$

\section{Conclusion}

Cette nouvelle approche constructive, consistant à traiter directement l'équation de Navier sans passer par une formulation variationnelle, présente de nombreux avantages. Elle permet notamment d'introduire (lors de l'adimensionnalisation de l'équation de Navier) des nombres sans dimension caractérisant la nature physique du problème. Ces nombres sont similaires à ceux introduits par J. Sanchez-Hubert et E. Sanchez-Palencia dans [21].

L'interprétation des nombres adimensionnels obtenus, nécessaire pour pouvoir ensuite les 
relier aux différentes puissances de $\varepsilon$, amène aux conclusions suivantes :

i) la donnée de l'ordre de grandeur des efforts extérieurs ou de façon équivalente de $\mathcal{F}_{\mathrm{t}}, \mathcal{F}_{3}, \mathcal{G}_{\mathrm{t}}$ et $\mathcal{G}_{3}$ en fonction de $\varepsilon$ détermine :

a) le rapport $\frac{u_{3 \mathrm{r}}}{V_{\mathrm{r}}}$

b) le modèle bidimensionnel final.

ii) pour des niveaux d'efforts extérieurs vérifiant $\mathcal{G}_{\mathrm{t}}=\varepsilon^{3}$ et $\mathcal{F}_{3}=\mathcal{G}_{3}=\varepsilon^{4}$, le rapport $\frac{u_{3 \mathrm{r}}}{V_{\mathrm{r}}}$ est égal à $\frac{1}{\varepsilon}$ et le modèle bidimensionnel obtenu est celuı de Kirchhoff-Love. La plupart des cas usuels de plaques minces en flexion relèvent de ces hypothèses.

On justifie ainsı pour ces niveaux d'efforts le décallage en $\varepsilon$ entre les composantes tangentılle et normale du déplacement, généralement pris comme hypothèse, aussı bien dans le cas linéaire $[4,6]$ que dans le cas non linéaire (modèle de Von Karman [5]).

Ainsi, le modèle de Kirchhoff-Love est valable pour des niveaux d'efforts où $\mathcal{F}_{3}=\frac{h f_{3 \mathrm{r}}}{\mu}$ et $\mathcal{G}_{3}=\frac{g_{3 \mathrm{r}}}{\mu}$ sont de l'ordre de $\left(\frac{h}{L}\right)^{4}\left(\mathcal{F}_{3}\right.$ et $\mathcal{G}_{3}$ étant des données du problème). Pour ces niveaux d'efforts, la déflexion est de l'ordre de l'épaisseur.

Notons enfin que même si cette approche, basée sur une formulation forte du problème est avant tout formelle, il est ensuite possible de revenir au cadre fonctionnel introduit par P.G. Ciarlet et P. Destuynder [4-6] pour retrouver les résultats classiques d'existence, d'unicité et de convergence.

\section{Bibliographie}

[1] Caillerie D., Thin elastic and periodic plates, Math. Methods in Appl. Scı. 6 (1984) 159191.

[2] Ciarlet P.G. et Destuynder P, Une justification du modèle bi-harmonique en théorie des plaques, C.R. Acad. Sci. Paris 285 (1977) 851-854.

[3] Ciarlet P.G. et Destuynder P., Une justification du modèle non linéaire en théone des plaques, C.R. Acad. Scr. Parzs 287 (1978) 33-36.

[4] Ciarlet P.G. et Destuynder P., Justification of the two-dimensional linear plate model, $J$. Mécanıque 18 (1979) 315-344.

[5] Ciarlet P.G, et Destuynder P., A justification of nonlinear model in plate theory, Comp. Meth. Appl. Mech. Engrg 17/18 (1979) 227-258.

[6] Ciarlet P.G., Plates and junctions in elastic multi-structures, An Asymptotic Analysis (Masson, Paris, 1990).

[7] Ciarlet P.G., Lods V. et Miara B., Analyse asymptotique des coques linéairement élastiques. I. Coques "membranaires", C.R. Acad. Scr. Parns 318 (1994) 863-868

[8] Ciarlet P.G., Lods V. et Miara B., Analyse asymptotique des coques linéairement élastiques. II. Coques "en flexion", C.R. Acad. Scr. Parrs 319 (1994) 95-100.

[9] Ciarlet P.G., Lods V. et Miara B., Analyse asymptotique des coques linéairement élastiques. III. Une justification du modèle de W. T. Koiter, C.R. Acad. Scr. Parns 319 (1994) 299-304. 
[10] Ciarlet P.G. et Lods V., Analyse asymptotique des coques linéairement élastiques. IV. Coques "membranaires sensitives", C.R. Acad. Scr. Parns 321 (1995) 649-654.

[11] Cimetière A., Sur la modélisation et le flambage des plaques élastoplastiques, Thèse d'état, université de Poitiers, 1987.

[12] Davet J L., Justification de modèles de plaques non linéarres pour des lois de comportement générales, Modélisation Mathématique et Analyse Numérique, 20 (1986) 225-249.

[13] Destuynder P., Sur une justification des modèles de plaques et de coques par les méthodes asymptotiques, Thèse d'État, Université de Pierre et Marie Curie, Paris, 1980.

[14] Goldenveizer A.L., Derivation of an approximate theory of bending of a plate by the method of asymptotic integration of the equations of the theory of elasticity, Prikl. Math. Mech. 26 (1962) 668-686.

[15] Miara B., Justıfication des mises à l'échelle et des hypothèses sur les données dans l'analyse asymptotique des modèles bidimensionnels de plaques. I Le cas linéaire, C.R. Acad. Scr. Parzs 314 (1992) 687-690.

[16] Miara B., Justification of the asymptotic analysis of elastic plates, I. The linear case, Asymptotic Analyszs 9 (1994) 47-60.

[17] Raoult A., Analyse Mathématique de Quelques Modèles de Plaques et de Poutres Élastiques ou Élasto-Plastiques, Thèse d'État, Université de Pierre et Marie Curie, Paris, 1988.

[18] Sanchez-Palencia E., Statique et dynamique des coques minces. I Cas de flexion pure non inhibée, C.R. Acad. Sci. Parts 309 (1989) 411-417.

[19] Sanchez-Palencia E., Statique et dynamique des coques minces. I. Cas de flexion pure inhibée - Approximation membranaire, C.R. Acad. Scu. Parzs 309 (1989) 531-537.

[20] Sanchez-Palencia E., Passage à la limite de l'élasticité tridimensionnelle à la théorie asymptotique des coques minces, C.R. Acad. Scr. Parss 311 (1990) 909-916. .

[21] Sanchez-Hubert J. et Sanchez-Palencia E., Introduction alux méthodes asymptotiques et à l'homogénéisation (Masson, 1992).

[22] Sanchez-Palencia E., Coques élastiques minces peu courbées. Transition continue entre les théories de plaques et de coques, C.R. Acad. Scr. Parıs 318 (1994) 783-790 